\title{
Stand Alone Personal Digital Health Monitoring System based on Android OS
}

\author{
Kevin Paulson \\ Department of Electronics and Telecommunication \\ Fr. C. R. I. T, Vashi \\ Mumbai, India
}

\begin{abstract}
In this paper, a low cost, portable and compact digital health monitoring system has been proposed. In this system biological parameters such as Electrocardiogram, Body Temperature and Pulse rate are acquired and displayed on an Android device. The signals will be displayed with the help of a Bluetooth connection between the Android device and Raspberry pi. The signals will be transmitted in real time to the Android device. An Android app has also been designed which will be capable of handling numerous Android devices and at the same the same multiple devices can also be connected to the proposed system. The Raspberry pi can be configured as a server thereby increasing the capacity of the system. Also the app that has been designed is simple to understand and use and as such can be used by anyone possessing an Android device.
\end{abstract}

\section{General Terms}

Wireless transmission, wireless networks, dynamic.

\section{Keywords}

Android, Android app, Bluetooth, Raspberry pi, Real time

\section{INTRODUCTION}

In today's world majority of the people is suffering from one type of disease, disorder etc. Out of these cardiovascular diseases are the most lethal in nature and has claimed the lives of many people around the world in the last decade alone. Cardiac illnesses are on the rise every day and this is a serious issue that is being seen in both the developed and the developing countries around the world. According to the 'World Health Organization' cardiovascular diseases causes more than 17 million deaths in the world each year and is responsible for more than half of the deaths in the United States alone. Based on many factors such as lifestyle, environment and surroundings many people fall prey to cardiac illness and this number is predicted to go on increasing every year. There are a number of reasons and risk factors that contribute to heart diseases such as the age, gender, smoking, excessive alcohol consumption etc. [1]. Out of this the age is by far the most important factor contributing to cardiovascular diseases. According to a statistical data 82 per cent of the people who die of cardiovascular diseases are in the age group of 65 years and above. Based on recent studies the risk of contracting a heart disease is increasing and it is has been seen that the age group of 30-40 years are more prone to cardiac diseases. Also men are at a greater risk of contracting heart diseases than women in the same age group. Also among the middle aged people cardiac illness is found 25 times more common in men than in women. Hence to monitor and prevent such kind of attacks in the future many new advanced and sophisticated techniques are being developed for early detection and recognition of cardiac illnesses. These techniques have been developed keeping in mind to provide the best possible diagnosis with the least cost

\author{
Jatin Desai \\ Department of Electronics and Telecommunication \\ Fr. C. R. I. T, Vashi \\ Mumbai, India
}

to everyone so that everyone's life can be saved which will be helpful in both the developing and the developed countries. Detecting a cardiac illness is of the most utmost importance since there are many types and it's crucial that one must identify the correct cardiac illness and administer the proper treatment accordingly. There are a number of reasons as to why the cardiac illnesses are not being identified early and even if they are identified majority of the time the patient is unable to receive the timely treatment which ultimately leads to more complications and finally results in death. Some of the reasons are as follows [2]:

- Precious time being lost while transporting the patient to the hospital

- Hospital or the clinic not having the up to date medical instruments or machines needed to identify or diagnose cardiac disorders

- Shortage of ECG treatments and unavailability of beds

- No early warning signs known to people

- Not enough qualified doctors available in rural areas

The Electrocardiogram (ECG) waveform is of crucial importance because a minor change in a person's ECG waveform is an indication that the person maybe suffering from one or other form of heart diseases. Hence it is necessary to display a real time ECG signal for better diagnostic purposes. However to display the ECG waveform on an Android device many factors need to be considered such as the background noise, processor speed, capability, distortions and disturbances. Also the system must be light, compact, portable and user compatible so that the person can see his/her waveform with ease and without having to worry about any technical details.

\section{RELATED WORK}

The earliest technique of displaying an ECG on a mobile phone was done using the Personal Digital Assistant (PDA). The PDA was popular due to the fact that it had many more features than a normal mobile phone and hence was ideal for displaying the ECG signal. Also the memory and the power of the PDA were superior to any other phones and as such had the capacity to support this system. The analysis of the ECG signal was done through a specially designed PDA module of the LABVIEW program [3]. The system performed a number of analyses through the program developed and finally sent the ECG signal through the internet and finally to the PDA. This was a major step and a success as the ECG signal was successfully detected and sent over the internet to the doctor or the hospital. Thus anyone with a PDA could check his/her ECG and consult with the doctor and get the necessary diagnosis. However, only people who possessed a PDA were able to take advantage of this technology. In the next few years with the introduction of Bluetooth technology and many 
mobile devices supporting Bluetooth technology, an ECG system based on Bluetooth was designed [4] - [5]. Since the range of Bluetooth was short around 10 meters, the ECG signal could be directly shown to the doctor and the use of the internet was not needed which further reduced the complexity of the system. Also because of the high data rates the information could be exchanged much more quickly than before. Since Bluetooth was operating in the unlicensed Industrial, Scientific and Medical (ISM) band it could be connected to phones, laptops, PDA's and other portable equipment's. With the coming of Android into the market this system was taken a bit further due to the compatibility between Bluetooth and Android. The coming of Android ushered in a new age of phones called 'Smartphones' which had much more computing power than earlier phones. Since Android is free and open source, majority of the mobile devices on the market now a day's run on Android and thus everyone could afford an Android phone. Due to Android's openness, it's simplicity to use and it's compatibility with Bluetooth, it could be directly linked to Android. Hence the next step was to display the ECG signal directly on the Android screen [6]. For this an app had to be created which will directly be connected to the Bluetooth module and will display the waveform accordingly. Because the Smartphones have high processing power data acquisition can be done on a real time basis and as a result the signal could be shown in real time and at a much faster rate.

\section{WORKING OF THE HEART}

The heart is an amazing organ as it pumps blood continuously to each and every cell of the body. It does this process repeatedly for 24 hours a day on an average of 60-100 beats per minute (BPM). This rate varies from person to person depending upon many factors such as the age, sex, race, location, lifestyle etc. The electrical activity of the heart is characterized by the normal rhythmical contraction of ventricles and atria which is followed by the oxygenation of the body. This regular functioning of the heart is due to the electrical impulses that stimulate the muscular mass of the heart to contract and expand simultaneously. This continuous contraction and relaxation is what gives the heart the pumping characteristic. This sequence is initiated by a group of nerve cells called as the SinoAtrial (SA) node, resulting in polarization and depolarization of the cells of the heart. This pulse travels from the SA node through the surrounding cells of the heart and then to the AtrioVentricular (AV) node. The $\mathrm{AV}$ node acts as a gate that allows the atria to finish contraction before allowing the pulse to move on to the ventricles. Each atrium pumps blood to a corresponding ventricle to provide blood to the lungs. The left ventricle sourced by the left atrium is the chamber that pumps blood throughout the body. The heart beats in a regular, even rhythm during a normal heartbeat and if the heartbeat is irregular then it can indicate a variety of problems such as 'Arrhythmia' in which the heartbeat maybe too irregular or faster or slower than the normal. If the heartbeat is too fast (more than 100 beats per minute) then the condition is called as 'Tachycardia' and if the heartbeat is too slow (less than 60 beats per minute) then the condition is called as 'Bradycardia'. If a person is suffering from any of the above conditions then it will be reflected on the ECG waveform accordingly. A medical expert can easily identify the problem and take the necessary steps to prevent it. This contraction and relaxation of the heart produces the $\mathrm{P}, \mathrm{Q}, \mathrm{R}, \mathrm{S}, \mathrm{T}$ waveform. An illustration of how an ECG waveform is developed is as shown in Fig 1 below:

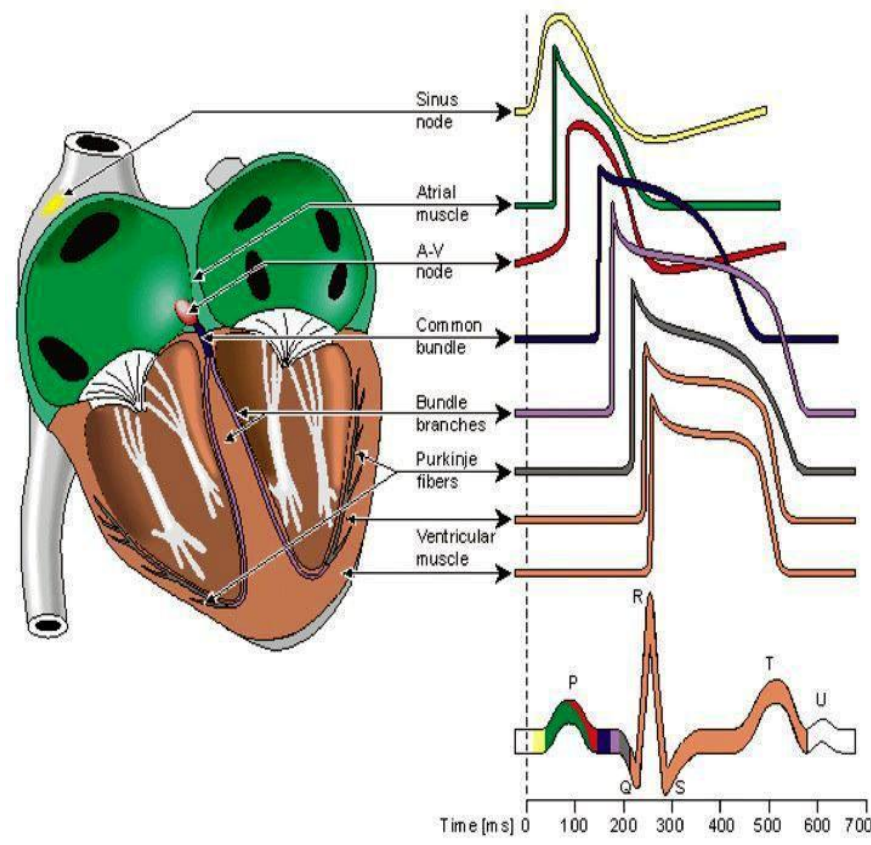

Fig 1: ECG waveform illustration

The names of the waves are $\mathrm{P}, \mathrm{Q}, \mathrm{R}, \mathrm{S}, \mathrm{T}$ because when 'Willem Einthoven' first viewed the ECG wave he assigned the letter P to the first wave due to the fact that it was a curve and also because he had studied the work of 'Rene Descartes' a French Philosopher and Mathematician who had invented analytical geometry and who had labelled some of the points on a curve as P and Q. Einthoven recognized this and thus named the first wave as $\mathrm{P}$ with the rest of the letters following sequentially as in the English Alphabet. Each of the individual $\mathrm{P}, \mathrm{Q}, \mathrm{R}, \mathrm{S}$, and $\mathrm{T}$ wave offers useful information about a person's heart condition [7]. Each eave is present for a specific interval of time and may vary in cases of persons suffering from any cardiovascular diseases. The ECG waveform begins with the $\mathrm{P}$ wave which is a small wave with duration of around of $80 \mathrm{~ms}$ which is caused by the atria contraction. This wave gives information about the total propagation time of the impulse to both the atria. A flat region is followed after the $\mathrm{P}$ wave known as the PR segment which is occurs due to the propagation of the electrical impulse from the atria to ventricles. This is then followed by the QRS complex which is characterized by the ventricular depolarization and it consists of a small Q wave, the high $\mathrm{R}$ wave and the small $\mathrm{S}$ wave. It gives useful information about the arrhythmias and fibrillations and can be very useful in diagnosing heart attacks. The ST wave that follows the QRS complex represents the period where the ventricles are contracting. Each segment and interval represents a particular aspect of the contraction and the relaxation of the heart. Hence if there are any changes in any of these segments and intervals it represents a particular cardiac problem that needs to be identified and treated immediately.

\section{HARDWARE IMPLEMENTATION}

The hardware section incorporates the ECG amplifier circuit which was designed and fabricated and whose output was observed on the oscilloscope. The ECG amplifier kit was designed using INA128P, LM358 and OPA2227. After many considerations it was decided that the circuit was to be designed using INA128P and OPA2227 because of the many advantages that OPA2227 had over LM358. There was noise present in the ECG signal due to many factors such as 
Electromagnetic Interference, Motion Artifacts, and Baseline wander and due to Respirations and Perspirations. The reason for choosing INA128 is because of its low offset voltage of $50 \mu \mathrm{V}$, Low Input Bias Current of 5nA, High Common Mode Ratio of $120 \mathrm{~dB}$ and wide supply range of \pm 2.25 to \pm $18 \mathrm{~V}$. The observed output of the ECG signal on the oscilloscope along with noise is as shown in the Figure 2 below:

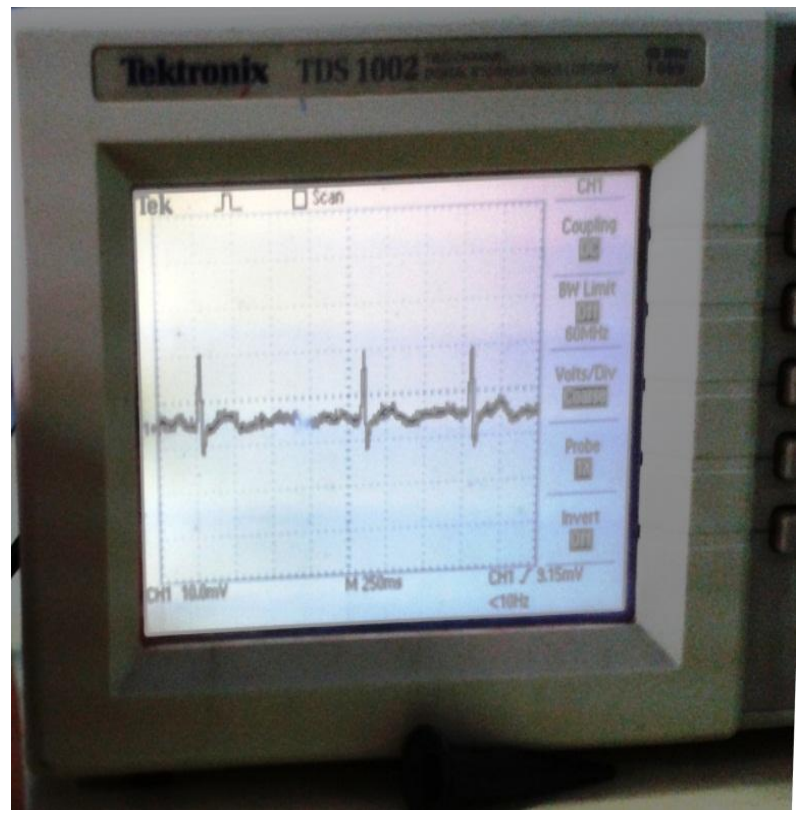

Fig 2: Observed noisy signal on the Oscilloscope

Since the main goal is to make the entire system light, compact and portable the proposed kit has been scaled down to size so that it can be easily transported anywhere. Surface Mounted Devices (SMD) resistors, capacitors and IC's were used because it occupies very less space and hence were advantageous for reducing the size of the kit. The input can be taken from the ECG leads that are connected to the body and the output given to the Arduino board. The output can be transmitted from the Arduino Board to the Android device with the help of the Bluetooth connection.

\section{SOFTWARE IMPLEMENTATION}

Since the main aim of this project is to develop a light, compact and portable health monitoring unit accordingly an Android app was also developed in par with the system. This app has been designed keeping in mind the latest versions and the updates of Android in the market so that it can support all the higher versions that will come out in the future [8]. The current version of Android is 4.4.2 also known as 'Kitkat'. The upcoming version is 'Lollipop' which is 5.0. The app must also support the lower versions up to 4.0 known as 'Ice Cream Sandwich' so that users with older Android versions will also be able to run this app in their mobile without facing any problems. The app has been designed in such a way that it is quick, simple and easy to understand so that anyone with an Android device will be able to take the benefit of it. The app is Bluetooth supported so that users can directly switch on the Bluetooth in their mobile phone and see the signal directly on the phone [9]. The app will also contain a database that will be linked to a server which will be hosted by the Raspberry pi. Raspberry pi is a small single sized computer specially developed for program purposes. It is primarily Linux based and has a number of operating systems on which it can work out of which 'Raspbian' is the recommended operating system for majority of the program applications. In this project also Raspbian was the operating system that was used. It can be configured in any way and so Raspberry pi was configured to act as a Bluetooth server so that a number of devices can connect to it at the same time. This increases the capacity of the system as a whole. The main advantage that this app has over the other health monitoring apps is that it is dynamic in nature and not static unlike others. There are other apps that are available in the market which are both paid and unpaid and the disadvantage of these apps is the fact that the cost of the paid apps are high and the unpaid ones rely on the personal details of the user thereby displaying an inaccurate version of their ECG. The app has been specifically designed to overcome both these drawbacks so as to provide a real time signal to the user whenever the user wants to see it. The logo and the name of the app are as shown below:

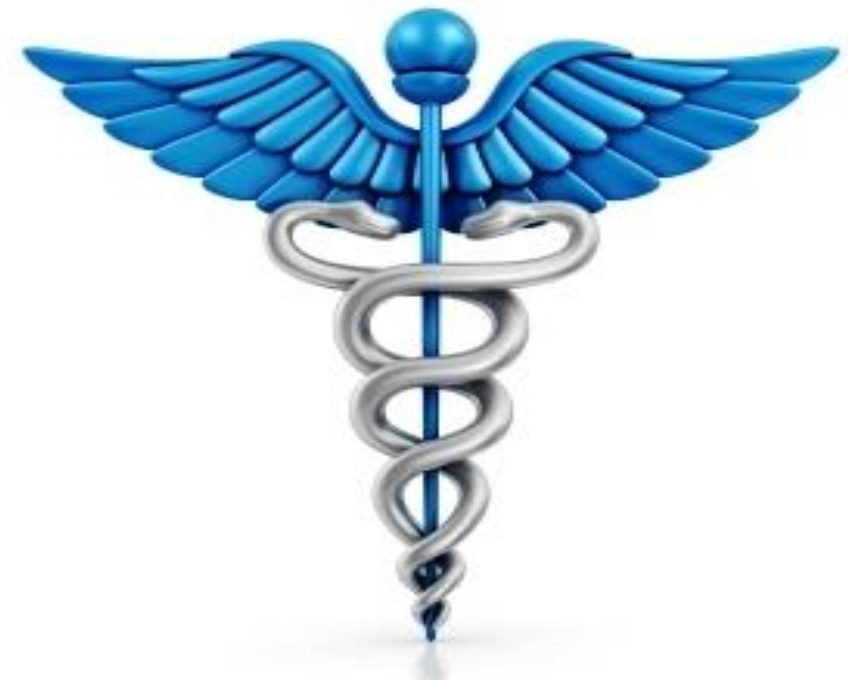

Fig 3: Digital Health Monitoring System

The app also contains a small database that stores the details of the patients such as Name of the patient, their address and contact no, name of the consulting doctor and contact no. This database will be hosted by the Raspberry pi which acts as a Bluetooth server as mentioned above. The programming was done in 'Eclipse' which is an Integrated Development Environment (IDE) mainly used for designing apps in Android. Along with Eclipse, the Android Development Tool (ADT) and the Software Development Kit (SDK) were also used for design, testing and launch purposes for developing the app [10]. All these tools are freely available on the internet and can be downloaded from the website of Android thus stemming the idea to make an app. This was not possible in any other mobile operating system due to the fact that Android is free and open source and hence anyone can download the tools and design an app of their choice. Other operating systems such as iOS, Windows are not open source and hence impossible for anyone to design an app in that platform. In Android there are two main types of files known as the JAVA file and the XML file. The JAVA file contains all the codes that are written for the functioning of the app, making the Bluetooth connections and other features while the

XML file contains the layout of the app such as the design and the layout as to how the app looks on an Android device. Both these files are essential in developing, designing and testing the app [11]. There is a graphical layout in Android where there are simple drag and drop widgets such as buttons, checkboxes, spinners, action bars, Text Views etc. which needs to be simply dragged and dropped down on a simple 
layout of an Android screen. The running and testing of the apps can be checked on both the Android Emulator and on the mobile device by simply connecting the mobile device to the desktop or the laptop via the Universal Serial Bus (USB) cable. The first view of the app is as shown below:

\section{Digital Health Monitoring Syst..}

Name of Patient

Contact No

Address of Patient

\section{Consulting Doctor}

\section{Contact No}

\section{Save and Proceed}

Fig 4: Patient Database

Figure 4 shows the patient database that can be seen in the app. The user has to enter the details and then click on the 'Save and proceed' button at the bottom of the screen. In Android there is a virtual table present that is similar to the SQLite table which is used for storing the data. Once the user clicks on the button he/she will be directed to the next page where the user can choose which parameter he/she wants to see from the dropdown menu. The dropdown menu is called as 'Spinner' in Android which is easy to use and store a large amount of items within itself as compared to the buttons which tend to populate and crowd the screen. In the next view the user can select from a list of parameters as what parameter the user wants to see. The database in Android has a small capacity and hence not suitable for handling large number of patients. This is the main reason why this database has to be hosted by the Raspberry pi. Raspberry pi has a memory capacity of 16 GB memory card which can be extended up to $32 \mathrm{~GB}$ which is advantageous for storing the database. Also if the more amount of memory is needed to be stored then one can go for higher memory sizes such as 64 GB to a small hard disk also to satisfy the memory requirements. The dropdown menu contains a list of parameters such as the ECG, Pulse rate and Body Temperature. The next view of the app shown in Figure 5 is as follows:
Displayparameters

Pulse Rate
Pulse Rate
Body Temperature
ECG
All

Fig 5: Parameters that are displayed

As mentioned above the user has to select any one of the parameters from the Spinner and the corresponding parameter will be displayed in real time. Here it should be noted that the Pulse Rate and the Body temperature circuits have not yet been developed. Once all the three circuits have been developed they can be mounted on top of one another so that it becomes compact in size due to the use of SMD materials being used as mentioned above.

\section{CONCLUSION}

Thus, a stand-alone digital health monitoring system has been proposed in this paper. The main aim of this paper is to provide a simple, dynamic and cost effective solution to the people so as to monitor their daily health parameters and provide them with real time and accurate readings. Moreover since the app is being developed on Android it will be beneficial to everyone who owns an Android device. As stated before the Body temperature and Pulse Rate circuits have not been developed and in addition to the above parameters many other parameters can also be included thus making it truly an effective personal digital health monitoring system for everyone.

\section{FUTURE SCOPE}

The following system can be modified to include many other biological parameters in addition to the three parameters mentioned above. The Raspberry pi attached to the board will act as the intermediary link between the board and the Android device. It will send the signal from the Arduino board to the Android device via a Bluetooth connection and the signal will be sent in real time so as long as the Bluetooth connection is made available the user can see his/her signal and once the Bluetooth connection is switched off, Raspberry pi stops sending the signal. As mentioned earlier the Raspberry pi can be configured as a Bluetooth server so that 
many devices can connect to it at the same time. This will be useful in ambulatory services on an outpatient basis where the patient is not hospitalized and still the treatment can be provided without the need of a formal fully hospital admission or the need for an overnight stay at the hospital. This is advantageous in the true sense that the medication or the surgery can be performed in a surgeon's office rather than in a hospital's operating room. Also it is most suited for healthy patients who have minor problems for which they do not have to travel all the way to the hospital for the treatment. Also in addition for the system to be configured as a Bluetooth server it can also be configured to act as a Web server. This increases the range, capacity and the capability of the system as compared to a Bluetooth server. Once configured as a Web server it can be used as a small LAN inside a hospital so that the doctor can check on any of his/her patients anytime of the day. Alert and emergency messages can also be included to alert the doctor to a particular patient when their vital stats are changing [12]. Since this app is being developed in Android a doctor with an Android phone can easily access the entire database and check on any of his patients even from their home as it will be connected through Wi-Fi. Moreover the database i.e. the first view of the app can also be expanded to include many more details concerning the patient. The increase in the patient database to include other vital information can be taken care of Raspberry pi as mentioned above. A hard disk can also be attached if necessary for storing the database. A centralized system can be setup in the hospital for monitoring all the patient that are admitted and also the patients who are at their home with their own personal system which will be linked to the hospitals centralized system via Wi-Fi or through a wired connection. A Special Emergency Distress and Service (SEDS) can also be implemented which will be fully dedicated to the database and if any emergency with any of the patient arises it will automatically alert the hospital's ambulance service so that it can quickly reach the patient's home and escort the patient back to the hospital as quickly as possible. The following Figure 6 highlights the future applications of the project [13]:

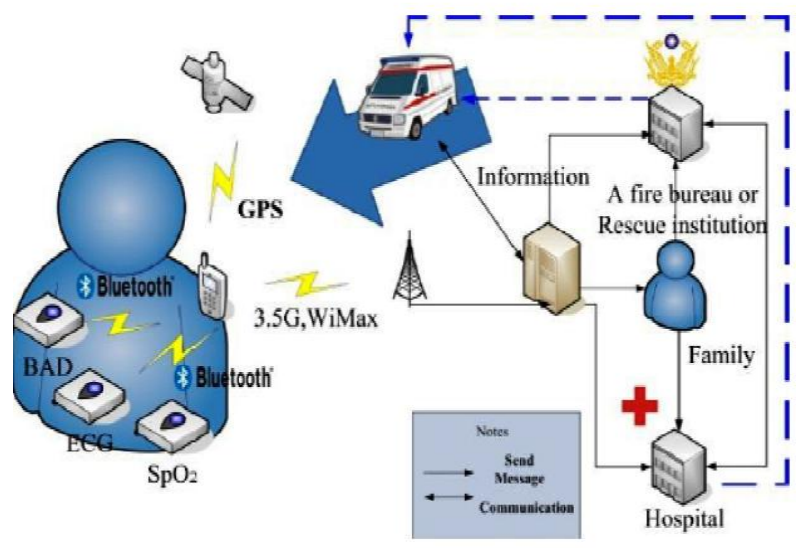

Fig 6: Future applications of the system

\section{REFERENCES}

[1] Dongdong Lou, Xiangxiang Chen, Zhan Zhao, Yundong Xuan, Zhihong Xu, Huan Jin, Xingzu Guo, Zhen Fang, "A Wireless Health Monitoring System based on Android Operating System", 2013 International Conference on Electronic Engineering and Computer Science.
[2] F. Belloni, D. Della Giustina, M. Riva, M. Malcangi, “A New Digital Stethoscope with Environmental Noise Cancellation", Dipartimento di Fisica,Universita degli Studi di Milano, Via Celoria 16, 20133 Milano ITALY.

[3] Claudio De Capua, Member, IEEE, Antonella Meduri, and Rosario, Student Member, IEEE, "A Smart ECG Measurement System Based on Web-Service-Oriented Architecture for Telemedicine Applications", IEEE transactions on Instrumentation and Measurement, VOL. 59, NO. 10, OCTOBER 2010

[4] J. Yepes, J. Aguirre, S. Villa, "Design of biomedical signal acquisition equipment with real-time constraints using Android platform", ARTICA, University of Antioquia, Medellin, Antioquia, Colombia.

[5] Gurpal Singh, Inderpal Singh, "Android OS Based Wireless Data Acquisition System via Bluetooth", International Journal of Research in Engineering and Technology.

[6] Ayaz Akram, Raheel Javed, Awais Ahmad, "Android Based ECG Monitoring System", Department of Electrical Engineering, University of Engineering and Technology Lahore, Pakistan, International Journal of Science and Research (IJSR), ISSN (Online): 2319-7064.

[7] http://circ.ahajournals.org/content/98/18/1937.full

[8] Patrick O. Bobbie, Chaudary Zeeshan Arif, Hema Chaudhari, Sagar Pujari, "Electrocardiogram (EKG) Data Acquisition and Wireless Transmission", Southern Polytechnic State University, School of Software Engineering, 1100 S. Marietta Parkway. Marietta, GA 30060, USA.

[9] Rajmane Sainath Bhagwanrao, Bahir Rahul Shivaji, Jeughale Shivam Workbench System", International Journal of Emerging Technology and Advanced Engineering, Volume 4, Issue 3, March 2014.Sadashiv, Prof.Aher Vishal A, "Android Based Wireless Electronics

[10] Jithin Krishnan, Niranjan D. Khambete, Biju B, “A Real time Data Acquisition and Monitoring Device for Medical Applications based on Android Platform", International Journal of Advanced Computer Research, Volume-3 Number-3 Issue-12 September-2013.

[11] Robert Sowah, Joana Nkrumah-Buadu, Seth Y. Fiawoo, "Design and Development of a Personal Health Monitoring System on Android Mobile Platform", International Journal of Engineering Science and Technology.

[12] Dhayal Bhatt, Tarulatta Chauhan, " A Bluetooth Enabled Personal Health Monitoring System Using Android Device”, IJEDR, Volume 2, Issue 2.

[13] Chin-Sung Wang, Chien-Wei Liu, Teing-Hui Wang, "A Remote Health Care System Combining a Fall Down Alarm and Biomedical Signal Monitor System in an Android Smart-Phone", International Journal of Advanced Computer Science and Applications", Vol.4, No.9, 2013 